\title{
BMJ Open Quality Improving medical residents' utilisation of integrated mental health in primary care
}

\author{
Jenna Palladino, ${ }^{1}$ Deirdra Frum-Vassallo, ${ }^{2}$ Joanne D Taylor, ${ }^{2}$ Victoria L Webb ${ }^{3}$
}

To cite: Palladino J, FrumVassallo D, Taylor JD, et al. Improving medical residents' utilisation of integrated mental health in primary care. BMJ Open Quality 2021:10:e001388. doi:10.1136/ bmjoq-2021-001388

- Additional supplemental material is published online only. To view, please visit the journal online (http://dx.doi.org/10. 1136/bmjoq-2021-001388).

Received 2 February 2021 Accepted 11 August 2021

\section{Check for updates}

(C) Author(s) (or their employer(s)) 2021. Re-use permitted under CC BY-NC. No commercial re-use. See rights and permissions. Published by BMJ.

${ }^{1}$ Psychiatry and Behavioral Health, Stony Brook University, Commack, New York, USA

${ }^{2}$ Psychology Service, Northport VA Medical Center, Northport, New York, USA

${ }^{3}$ Main Line Therapy and Psychological Services, LLC, Wayne, Pennsylvania, USA

Correspondence to Dr Jenna Palladino; jenna.palladino@ stonybrookmedicine.edu

\section{ABSTRACT}

Background Integration of mental health services allows for improved prevention and management of chronic conditions within the primary care setting. This quality improvement project aimed to increase adherence to and functioning of an integrated care model within a patientcentred medical home. Specifically, the project focused on improving collaboration between Primary Care Mental Health Integration (PC-MHI) and the medical resident Patient Aligned Care Teams (PACT) at a Veterans Affairs Medical Center in Northport,New York (VAMC Northport). Method The project used increased education, training and relationship building among the medical resident PACTs, and the establishment of regularly occurring integrated team meetings for medical and mental health providers. Education of residents was measured with a self-assessment pre-training and post-training, while utilisation was measured by the percentage of patients currently on a PACT's panel with at least one PC-MHI encounter in the last 12 months (known in VAMC Northport as PACT-15 metric).

Results Two resident PACTs that received both training and weekly integrated meetings increased their utilisation of integrated mental health services by $3.8 \%$ and $4.5 \%$, respectively. PACTs that participated in training only, with no regular meetings, showed an initial improvement in utilisation that declined over time.

Conclusions Training alone appeared beneficial but insufficient for increased integration over time. The addition of a regularly occurring integrated weekly meeting may be a critical component of facilitating sustained mental health integration in a primary care medical home model.

\section{INTRODUCTION \\ Problem description}

The Veteran's Health Administration (VHA) embraces a medical home model of care, known as the Patient Aligned Care Team (PACT) initiative. ${ }^{1}$ This model emphasises team-based, collaborative, patient-centred care. A primary care PACT is composed of a core (physician, registered nurse care manager, licensed practical nurse or medical assistant, and administrative clerk) and expanded (eg, nutrition, social work, clinical pharmacy, mental health) team. ${ }^{2}$ The Primary Care Mental Health Integration (PC-MHI) service provides mental health interventions within PACTs. Colocated, collaborative PC-MHI providers are available to conduct a variety of indirect and direct patient care activities, including warm handoffs, behavioural and psychopharmacological consultation, brief treatment of mental health conditions and assistance with chronic disease management. Treatment planning is collaborative across PACT members to facilitate the development of a joint treatment plan.

At the Northport Veterans Affairs Medical Center (VAMC Northport), PC-MHI was available to provide the aforementioned services; however, integration was suboptimal and inconsistent. This was evidenced by low utilisation across PACTs, with the lowest rates found among the PACTs consisting of medical residents. Utilisation includes direct patient care services as well as indirect patient care services (eg, consultation).

\section{Available knowledge}

Integration is central to the VA's proactive patient-centred PACT model. ${ }^{1}$ Integrated care refers to multidisciplinary care that includes patient involvement and collaboration across disciplines. The integration of primary care and mental health services allows for service coordination across specialties to provide preventive and curative care. ${ }^{3}$ Consistent utilisation of PC-MHI facilitates coordination of care and development of treatment plans focused on overall well-being. ${ }^{2}$ Such integration allows for better prevention and management of chronic conditions within the primary care setting. ${ }^{4}$ Chronic conditions (eg, diabetes, hypertension and congestive heart failure) are the leading cause of mortality in the world, accounting for approximately $63 \%$ of all deaths. ${ }^{5}$ Within the USA, chronic diseases affect approximately 133 million people and lead to seven out of ten deaths each year. ${ }^{5}$ Additionally, $75 \%$ of all healthcare costs are due to chronic health conditions. ${ }^{5}$ Lifestyle changes are critical for managing and preventing chronic conditions. ${ }^{5}$ Intensive behavioural interventions assist patients 
in effectively initiating and maintaining these lifestyle changes. ${ }^{6}$ In the VHA, PC-MHI teams are well positioned to provide these interventions to primary care patients.

In addition to health promotion and disease prevention, PC-MHI utilisation can provide other benefits. Integrated mental healthcare can improve access and increase the likelihood that patients will follow through with mental health treatment. ${ }^{7}$ Patients are more likely to fulfil a mental health appointment when it takes place in a primary care setting. ${ }^{8}$ Integrated mental healthcare may also reduce mental health stigma, ${ }^{79}$ improve providers' knowledge and confidence ${ }^{10} 11$ and be more cost-effective. ${ }^{12}{ }^{13}$ Trials of these models demonstrate additional impacts including improved treatment adherence and greater increases in functioning. ${ }^{1415}$

\section{Rationale}

One approach to improving integration is to provide additional opportunities for communication between primary care and mental health providers. Frequent communication is critical for collaboration, and allows for knowledge sharing, shared decision making and goal setting. ${ }^{16}$ Past research shows that comanagement of patients between primary care and mental health providers leads to increased self-reported knowledge and educational benefit from residents in a family medicine programme. ${ }^{10}$ Care providers can benefit from training in mental health treatment and the behavioural aspects of chronic disease management, and such training is advocated by the American College of Physicians. ${ }^{7}$ Residents in particular report improved confidence and learning after collaborating with integrated mental health providers. ${ }^{10}$ As such, this project aimed to provide training to a subset of PACTs (those with medical residents) and to create a forum for ongoing communication and consultation through a weekly meeting. An expected outcome of these efforts was improved utilisation of PC-MHI by these PACTs.

\section{Specific aims}

The initial aim of this project was to better understand and contribute to medical residents' knowledge of the PACT model, PC-MHI and utilisation strategies. Pursuit of this aim led to two additional aims: (1) establish regularly scheduled integrated team meetings within Internal Medicine resident PACTs and (2) increase the utilisation of PC-MHI by resident PACTs.

\section{METHODS AND RESULTS \\ Context}

This project was conducted over the course of fiscal year 2018. Primary care at the medical centre saw on average about 25000 unique patients per month. Approximately 20000 patients were classified as urban and approximately 500 as rural. Comorbidities were high in rates of obesity (6996 patients), cardiovascular disease, chronic obstructive pulmonary disease, diabetes and hypertension. Approximately 3000 patients held the diagnosis of PTSD and 2700 held the diagnosis of depression. Women
Veterans made up an average of $7.5 \%$ of team assignments. The average panel size was around 840 patients. Patients were assigned to teams randomly based on availability and overall panel size. Administrative staff would assign new Veterans to panels ensuring for consistency in patient demographics across panels. Leadership reviewed the overall makeup of each PACT and found comparable demographics across the medical system (eg, \% of Veterans with various levels of complexity or comorbid conditions, age ranges). There were no intended differences in the panels.

Barriers to improved integration existed at the start of this project. First, resident PACTs did not hold regular integrated team meetings (eg, those involving expanded team members) and demonstrated low utilisation rates of PC-MHI. Medical residents' time was subject to multiple demands, including broader medical training needs and responsibilities on inpatient floors. Additionally, residents at VAMC Northport worked within a PACT only once every 5 weeks (known as a $4+1$ model), leading to limited awareness of the PACT model and ways in which PC-MHI could be used in this facility.

However, promising opportunities to improve integration were also present at the start of the project. Historically, resident PACTs were distinct from other primary care PACTs because they were aligned under the Department of Medicine (rather than primary care). At the start of this project, all PACTs were reassigned under the Department of Medicine, consolidating the leadership and allowing opportunity for improved integration. Additionally, VHA began emphasising the importance of PC-MHI utilisation around the time of this project's initiation. These changes in institutional structure highlight leadership's support of further integration of mental health services within PACT. Institutional leadership also prioritised these integration efforts by allowing clinic time to be blocked for the interventions discussed in this project.

Due to the aforementioned unique challenges and opportunities within resident PACTs, this project specifically focused on improving integration within these teams. There were a total of six residents PACTs that were targeted for this project. Resident PACTs consisted of Programme Year (PGY) 1-4 residents. Efforts were made to equally divide the number of residents in each PGY across resident PACTs. As noted above, there were no intended differences between patients on resident PACT panels versus patients on other PACT panels across the medical centre.

To help quantify integration, this project tracked a specific pre-existing VHA metric measuring PC-MHI utilisation (PACT 15 metric). The PACT 15 metric measured the percentage of patients currently on a PACT's panel who had at least one encounter with PC-MHI during the last 12 months. The target for this metric was set at $10 \%$ for all PACTs. At the start of this project, the metric was below target for five of the six resident PACTs. A specific aim of this project was to improve the PACT 15 metric for each resident PACT team by $1 \%$. Authors used 
established changes in PACT 15 data from 2 years prior to the onset of this project to develop this aim. Time series data are not available for the identified Resident PACTs prior to the onset of this project. PACT 15 changes within the VHA nationwide were $0.37 \%$ from December 2015 to December 2016 and $0.84 \%$ from December 2016 to December 2017. PACT 15 changes within the medical centre were $0.78 \%$ from December 2015 to December 2016 and 1.83\% from December 2016 to December 2017. To accomplish this aim, two Plan-Do-Study-Act (PDSA) cycles were conducted in the course of this project. ${ }^{17}$

The interventions used in this project were not part of a budget and no funding was specially allocated for it. However, the postdoctoral fellowship (within which this project was conducted) is a paid position through the Office of Academic Affiliations.

\section{PDSA cycle 1: intervention}

The 'Plan' phase of the first PDSA cycle aimed to gather more information on the nature of the problem and the needs of invested stakeholders. During this phase (December 2017), meetings of relevant stakeholders (chief of medicine, chief of primary care, director of medical resident training, and the chief medical resident) were held to secure leadership support and gain information about interventions needed to address the discrepancy between the current state and goal state. The goal was to better understand possible barriers to resident PACTs embracing the PACT model and using PC-MHI. Further training and education for the residents was a key need that emerged.

The 'Do' phase of the first PDSA cycle focused on providing training on this model to the residents (January-February 2018). A 90 min training was developed and provided to groups of residents over 5 weeks (to reach all rotating residents in primary care) (online supplemental appendix $\mathrm{C}$ ). This training occurred during a pre-established meeting time for resident groups, which allowed for the training to be implemented rapidly after agreement of key stakeholders. All resident PACTs attended this intervention. The training focused on: (1) the overall PACT model, (2) the role of PC-MHI in chronic disease management, personalised health planning and mental health treatment and (3) opportunities and strategies for utilising PC-MHI. The training also allowed residents to meet PC-MHI staff and ask questions about the model.

\section{PDSA cycle 1: study of the intervention and measures}

The effectiveness of training efforts was measured by selfassessments of residents' knowledge, completed before and after the 90 min training. Both the premeasure and postmeasure assessed residents' knowledge of PACT, PC-MHI, the function of these services, utilisation strategies and staff alignment with eight 5-point Likert scale items (disagree to agree) and one open-ended item (see online supplemental appendix A and B).
PDSA cycle 1: analysis and results

In the 'Study' phase of the initial PDSA cycle, residents' responses on the pre-self-assessments and post-selfassessments were averaged and open-ended responses were coded for themes (February 2018). Prior to the training, residents disagreed or somewhat disagreed that they had knowledge of the PACT model, PC-MHI and utilisation strategies. This disagreement was consistent across all eight items administered on this questionnaire. After the training, residents agreed or somewhat agreed that they had knowledge of the PACT model, PC-MHI and utilisation strategies. This agreement was consistent across all eight items administered on this questionnaire. Due to the anonymity of premeasures and postmeasures, we were unable to run a conventional test of repeated measures (preassessment and postassessment data could not be linked). As an alternative approach for testing the hypothesis of change, we conducted a one-sample t-test to compare participants' postassessment responses against the grand mean of all participants' preassessment responses. There was a significant difference found in participants' postassessment scores when compared with the grand mean of preassessment scores, for all self-report items (question 1: $(\mathrm{t}(29)=29.66, \mathrm{p}<0.001)$; question 2: $(\mathrm{t}(29)=29.15 .77, \mathrm{p}<0.001) ;$ question 3: $(\mathrm{t}(29)=24.44$, $\mathrm{p}<0.001)$; question 4: $(\mathrm{t}(29)=20.64, \mathrm{p}<0.001)$; question 5: $\quad(\mathrm{t}(29)=16.63, \mathrm{p}<0.001) ; \quad$ (question $6: \quad(\mathrm{t}(29)=16.10$, $\mathrm{p}<0.001)$; question 7: $(\mathrm{t}(29)=31.72, \mathrm{p}<0.001)$; question 8: $(\mathrm{t}(29)=31.23, \mathrm{p}<0.001)$. See table 1 for average responses to questionnaire items on premeasures and postmeasures.

\section{PDSA cycle 1: discussion}

In the 'Act' phase of the first PDSA cycle, results indicated a consistent improvement in self-reported understanding and knowledge of PACT model, PC-MHI and utilisation strategies. Residents expressed a strong interest in the PC-MHI/PACT integration model and were interested in working more closely with the PC-MHI team.

\section{PDSA cycle 2: intervention}

In the 'Plan' phase of the second PDSA cycle, a review of other PACTs at Northport VAMC was conducted to identify factors that improved integration (March 2018). This review demonstrated the value of regular team meetings in increasing PC-MHI utilisation. As such, we aimed to establish regular integrated resident PACT meetings in order to continue contributing to resident PACT utilisation of PC-MHI. Establishment of these meetings was also consistent with the preferences expressed by residents during their trainings.

In the 'Do' phase of the second PDSA cycle, regular integrated team meetings were piloted with two of the six resident PACTs (intervention began on April 2018). These meetings were scheduled for 1 hour to allow for adequate teaching time for residents during the integration meetings. Project piloted this PDSA cycle with two of the six resident PACTs due to staffing limitations 
Table 1 Average Responses to Questionnaire Items on Premeasures and Postmeasures

\begin{tabular}{lll}
\hline Question & Preaverage & Postaverage \\
\hline $\begin{array}{l}\text { 1. I feel confident that I } \\
\text { know what a PACT team } \\
\text { stands for. }\end{array}$ & 2.71 & 4.84 \\
$\begin{array}{l}\text { 2. I feel confident that I } \\
\text { know how a PACT team } \\
\text { functions. }\end{array}$ & 2.68 & 4.65 \\
\end{tabular}

$\begin{aligned} & \text { 3. I feel confident that I } \\ & \text { know how the members of }\end{aligned}$
a PACT team are.
$\begin{aligned} & \text { } \text {. I feel confident that I } \\ & \text { know what the function of }\end{aligned}$
PCMH is.
$\begin{aligned} & \text { 5. I feel confident that } \\ & \text { I know when to utilize }\end{aligned}$
PCMH.
$\begin{aligned} & \text { 6. I feel confident that I } \\ & \text { know who the PCMH staff } \\ & \text { aligned with my team is. }\end{aligned}$
$\begin{aligned} & \text { 7. I am aware of how to } \\ & \text { refer a patient to PCMH for }\end{aligned}$
a same day appointment.
$\begin{aligned} & \text { 8. I am aware of how to } \\ & \text { refer a patient to PCMH }\end{aligned}$
when the patient is not
available for a same day
appointment.

PACT, Patient Aligned Care Team; PCMH, Primary Care Mental Health.

within PC-MHI and time limitations of a rapid quality improvement project. Resident PACTs 5 and 6 were identified to receive the team meeting intervention based on their schedules aligning with staff availability. Initially, meetings included PC-MHI staff, attending physicians, residents, Registered Nurses (RNs) and the medical staff clerk. Meetings were focused on team building, consultation, identification of patients for follow-up and panel management (proactively planning for upcoming patient visits).

\section{PDSA cycle 2: study of the intervention and measures}

In cycle 2, the frequency of integrated resident PACT meetings and the utilisation of PC-MHI by resident PACTs were assessed (June 2018). The latter was measured by the PACT 15 metric (as noted above, a measure of the percentage of patients currently on the primary care panel who have had at least one encounter with PC-MHI during the last 12 months). The PACT 15 metric for resident PACTs 1-4 (those who did not participate in ongoing weekly meetings) was compared with resident PACTs 5 and 6 (the two teams who participated in ongoing weekly meetings).
Table 2 PACT 15 Data from December 2017 to May 2018

December May 2018 2017 PACT 15 PACT 15 Difference

\begin{tabular}{lrrc}
\hline All VHA & $8.1 \%$ & $8.3 \%$ & 0.2 \\
\hline 1V02 & $8.2 \%$ & $8.5 \%$ & 0.3 \\
$\begin{array}{l}\text { Northport, NY } \\
\text { Healthcare System }\end{array}$ & $7.3 \%$ & $8.1 \%$ & 0.8 \\
\hline Northport, NY & $8.4 \%$ & $9.7 \%$ & 1.3 \\
\hline RESIDENT PACT 1 & $2.3 \%$ & $1.9 \%$ & -0.4 \\
\hline RESIDENT PACT 2 & $4.6 \%$ & $5.7 \%$ & 1.1 \\
\hline RESIDENT PACT 3 & $4.8 \%$ & $5.7 \%$ & 0.9 \\
\hline RESIDENT PACT 4 & $1.8 \%$ & $2.1 \%$ & 0.3 \\
\hline RESIDENT PACT 5 & $3.3 \%$ & $7.8 \%$ & 4.5 \\
\hline RESIDENT PACT 6 & $10.2 \%$ & $14.0 \%$ & 3.8 \\
\hline
\end{tabular}

PACT, Patient Aligned Care Teams; VHA, Veteran's Health Administration.

\section{PDSA cycle 2: analysis and results}

In the 'Study' phase of the second PDSA cycle, an increase in consistent integrated team meetings was observed for the two resident PACTs participating in the pilot. These teams held 15 team meetings in 17 weeks, whereas the four teams not participating in the pilot did not hold any integrated meetings. Additionally, the two resident PACTs that participated in both the resident training and the weekly PACT meetings saw an increase in the PACT 15 of $3.8 \%$ and $4.5 \%$, respectively. This improvement in the PACT 15 metric also outpaced that of the average increase in other PACTs within both the Northport VAMC and neighbouring VAMCs, which improved by $1 \%$ and $.2 \%$, respectively (see table 2 ). In comparison, the four resident PACTs that participated in only the training (ie, no ongoing weekly meeting) showed an initial improvement in their PACT 15 metric that then slowly declined over time. For example, Resident PACT 1 had an initial PACT 15 rate of $2.31 \%$, which nominally improved to $2.35 \%$ a month after the training. Without the addition of the weekly team meetings, the PACT 15 rate for this team returned to below baseline $(1.88 \%)$. PACT15 rates are also provided in line graph form on figure 1 . Numerators and denominators for PACT 15 data were not available in the VA data set that was used for this project.

\section{PDSA cycle 2: discussion}

In the 'Act' phase of the second PDSA cycle, the results highlighted the importance of team meetings for improving utilisation of PC-MHI. The incorporation of additional training appears to be a key intervention to increasing resident understanding of integrated carePACT members appeared invested in team meetings and they consistently met weekly despite changes in staffing, residents and meeting location. This finding is consistent with previous research showing that direct feedback and coordination of care between primary care and mental health providers leads to reinforcement of residents' 


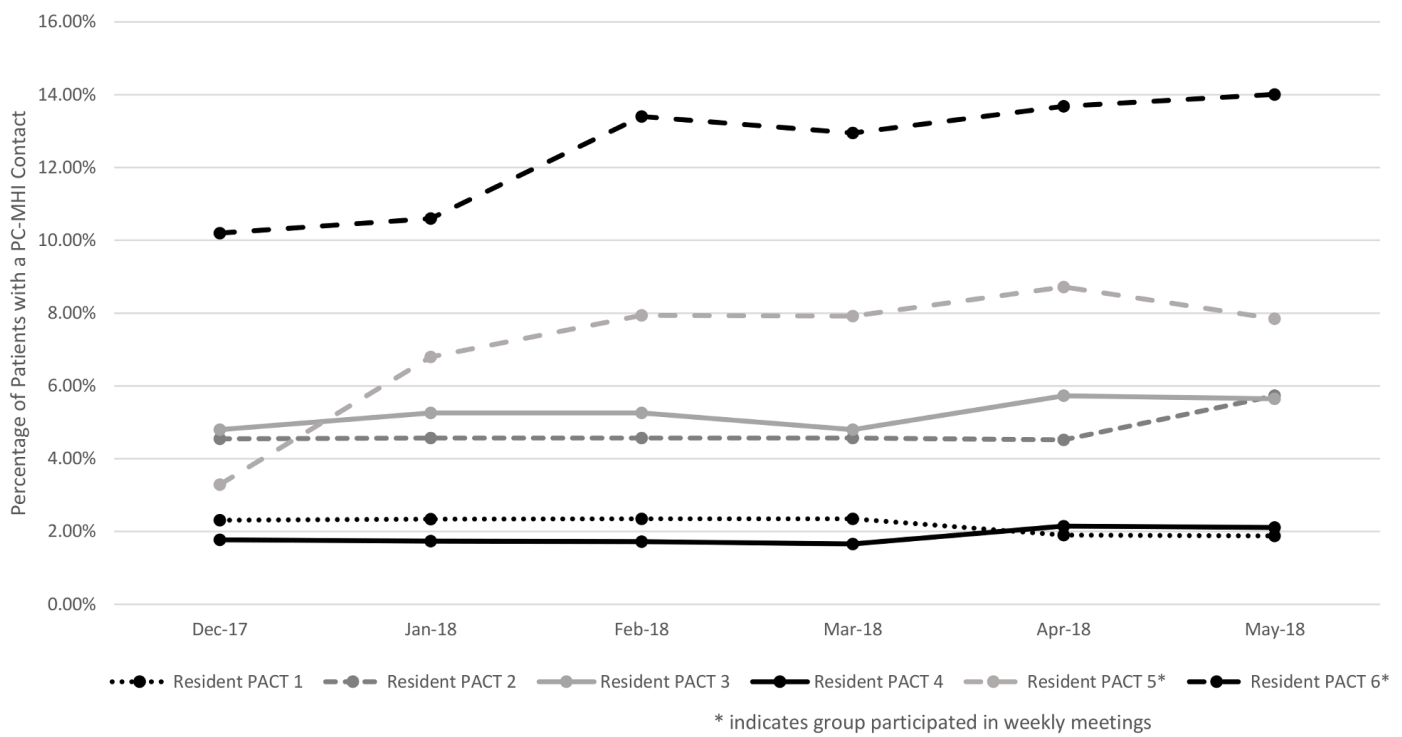

Figure 1 Percentage of patients with a PC-MHI contact. PACT, Patient Aligned Care Teams; PC-MHI, Primary Care Mental Health Integration.

acumen in working with multidisciplinary teams and improvements in care coordination. ${ }^{10}$

\section{GENERAL DISCUSSION}

\section{Summary and interpretation}

The findings of this quality improvement project highlight two important components for improving integration within a patient-centred primary care team. First, training medical residents in the collaborative, patientcentred model and educating them about the available collaborative, colocated mental health resources appeared to improve their knowledge and openness to both the model and resources. Residents actively participated in the weekly team meetings that occurred during the second PDSA cycle and there were indicators of continued resident knowledge present in these meetings (eg, proper identification of PC-MHI referrals, openness to consulting with PC-MHI team). Due to the complicated nature of resident training programmes, special attention should be placed on ensuring training of residents at the start of their programme with adequate follow-up training throughout residency. In this project, such an emphasis on training helped residents feel better equipped to use primary care mental health. As a result of this project, these trainings will continued for the upcoming resident classes and begin at the onset of their residency programme.

Critically, while resident PACTs that participated in only the training (ie, no ongoing weekly meeting) showed an initial improvement in utilisation of PC-MHI, this improvement then slowly declined over time. In contrast, the resident PACTs that received both the training and regular ongoing meetings demonstrated a sustained increase in their utilisation of PC-MHI. These teams also had a greater increase in utilisation than VAMC Northport and the neighbouring VAMCs. Thus, while training is an important intervention, it does not appear to be sufficient to sustain ongoing utilisation. Consistent, ongoing collaborative team meetings are likely a key component to improving integration. Weekly meetings allowed for team building, improved panel management, closer patient follow-up and overall interdisciplinary collaboration. At the conclusion of this project, the teams planned to continue to meet regularly with the goal of expanding to include all residents and increasing the length of the meeting time.

An additional takeaway from this project is the importance of leadership involvement. While buy-in from key stakeholders within the PACTs was vital to the success of the project, leadership support was especially critical. Medical providers have multiple time demands, making new initiatives difficult to implement. Leadership involvement allowed for the promotion of integration efforts through prioritisation of utilisation of PC-MHI services and allowing for clinic time to be blocked for team meetings.

\section{Challenges}

Due to a limitation in resources, the weekly team meetings were only offered to two resident PACTs. There is a possibility for selection bias given that the two resident PACTs identified for additional interventions were not chosen at random (instead, they were chosen based on scheduling availability). However, the authors believe the findings would be consistent across all PACTs offered this intervention due to the institutional support (ie, time carved out for meetings) and similar trends observed in subsequent quality improvement initiatives. The remaining four resident PACTs who did not receive ongoing meetings were used as a comparison group to evaluate for differences in clinically meaningful PACT 15 change. While this allowed for a better understanding of the importance of ongoing 
meetings versus a single training alone, without additional resources, it is unknown if the same findings would generalise across all teams.

An additional challenge that arose during this project was staffing. We observed that a higher level of integration produced higher levels of utilisation. At the time of this project, PC-MHI staff were not always available to meet with PACTs, despite increasing interest in and desire for integration across resident and non-resident PACTs. As a part of this project, attending physicians, internal medicine residents, PC-MHI staff psychologist, registered nurse aligned with the resident PACT and PC-MHI postdoctoral fellow attended the meeting weekly. When possible, the medical support assistants and social workers aligned with the resident PACT would attend. Increased staff support would provide the resources needed to help build and sustain team meetings, which were an essential component to integration and yielded increased utilisation. This project demonstrated the importance of ensuring that adequate PC-MHI staffing is established as integration increases.

A final limitation of this project relates to the inability to complete advanced statistical analyses due to the small sample size and constraints in available types of data. Additionally, due to staffing changes, a cross-over project cannot be completed. The authors believe that these findings were not wholly due to chance as coordination of care is a core component of integrated care programmes. ${ }^{2}$ The weekly team meetings allowed for continual collaboration and care coordination-residents became more familiar with PC-MHI staff and expressed enthusiasm for collaboration. During meetings, residents also demonstrated increasing ability to identify patients who would benefit from PC-MHI services. Additionally, even after this project concluded, meetings continued to run weekly with staff psychologist and postdoctoral fellow in attendance until remote work set in due to COVID-19. Following the conclusion of this project, the resident PACTs that received both the educational and meeting interventions continued to be highly integrated with PC-MHI using shared medical appointments frequently and referring to PC-MHI frequently. Additionally, subsequent quality improvement initiatives modelled after this project have occurred since its conclusion, demonstrating a similar approach has improved integration among various PACT teams across the medical centre. The authors believe that these continued efforts and associated improvements in integration are indication that collaborative efforts (such as weekly team meetings) meaningfully contribute to greater utilisation of PC-MHI services. Nonetheless, future research should aim to utilise random assignment, larger sample sizes and diverse data collection methods to more formally evaluate the impact of these interventions.

\section{CONCLUSIONS AND FUTURE DIRECTIONS}

Multiple interventions were incorporated into this project and, as alluded to above, continued efforts to sustain and further improve integration have been made since its conclusion. These steps thus far include continued weekly team meetings (eventually with more resident trainees) and continued trainings for residents (ideally to occur at the start of residency to provide more immediate education). Continued training on the PACT model and PC-MHI may be helpful at later points in the residency programme and should be considered as a future addition to this project. Additionally, ongoing establishment of regular meetings, trainings and other opportunities for collaboration across all primary care PACTs is likely to contribute to improved integration. Future research is needed to evaluate the impact of this improved integration on patient care.

Acknowledgements The authors wish to acknowledge Edward Weissman, MD, Gerald Cruise, MD, and Lisa Fisher, MD, and Cathy Cruise, MD, and Astrid Pilgrim, MD for their support of this project.

Contributors All authors were involved in the planning, coordination and submission of the project and were actively involved in the implementation of PDSA cycles.

Funding The authors have not declared a specific grant for this research from any funding agency in the public, commercial or not-for-profit sectors.

Competing interests None declared.

Patient consent for publication Not required.

Ethics approval The present project was reviewed by the chair of the institutional review board (IRB) at the Northport VAMC. The project was given a non-research declaration and was exempt from IRB approval.

Provenance and peer review Not commissioned; externally peer reviewed.

Data availability statement All data relevant to the study are included in the article or uploaded as supplementary information.

Supplemental material This content has been supplied by the author(s). It has not been vetted by BMJ Publishing Group Limited (BMJ) and may not have been peer-reviewed. Any opinions or recommendations discussed are solely those of the author(s) and are not endorsed by BMJ. BMJ disclaims all liability and responsibility arising from any reliance placed on the content. Where the content includes any translated material, BMJ does not warrant the accuracy and reliability of the translations (including but not limited to local regulations, clinical guidelines, terminology, drug names and drug dosages), and is not responsible for any error and/or omissions arising from translation and adaptation or otherwise.

Open access This is an open access article distributed in accordance with the Creative Commons Attribution Non Commercial (CC BY-NC 4.0) license, which permits others to distribute, remix, adapt, build upon this work non-commercially, and license their derivative works on different terms, provided the original work is properly cited, appropriate credit is given, any changes made indicated, and the use is non-commercial. See: http://creativecommons.org/licenses/by-nc/4.0/.

\section{REFERENCES}

1 Rosland A-M, Nelson K, Sun $\mathrm{H}$, et al. The patient-centered medical home in the Veterans health administration. Am J Manag Care 2013;19:e263-72.

2 Kearney LK, Post EP, Zeiss A, et al. The role of mental and behavioral health in the application of the patient-centered medical home in the Department of Veterans Affairs. Trans/ Behav Med 2011;1:624-8.

3 Valentijn PP, Pereira F, Sterner CW, et al. Validation of the rainbow model of integrated care measurement tools (RMIC-MTs) in renal care for patient and care providers. PLoS One 2019;14:e0222593.

4 Beehler GP, Funderburk JS, PR KJ. The role and functions of embedded behavioral health providers in VA primary care-mental health integration: current evidence and future directions for research. A VA center for integrated healthcare white paper. Center for Integrated Healthcare 2015.

5 Center for Disease Prevention and Health Promotion. Chronic disease prevention and health promotion, 2018. Available: http:// www.cdc.gov/chronicdisease/index.htm [Accessed 25 Jun 2018]. 
6 Newsom JT, Huguet N, McCarthy MJ, et al. Health behavior change following chronic illness in middle and later life. J Gerontol B Psychol Sci Soc Sci 2012;67:279-88.

7 Crowley RA, Kirschner N, Health and Public Policy Committee of the American College of Physicians. The integration of care for mental health, substance abuse, and other behavioral health conditions into primary care: Executive summary of an American College of physicians position paper. Ann Intern Med 2015;163:298-9.

8 Speer DC, Schneider MG. Mental health needs of older adults and primary care: opportunity for interdisciplinary geriatric team practice. Clin Psychol Sci Prac 2003;10:85-101.

9 Shim R, Rust G. Primary care, behavioral health, and public health: partners in reducing mental health stigma. Am J Public Health 2013;103:774-6.

10 Hemming P, Hewitt A, Gallo JJ, et al. Residents' confidence providing primary care with behavioral health integration. Fam Med 2017;49:361-8.

11 Zallman L, Joseph R, O'Brien C, et al. Does behavioral health integration improve primary care providers' perceptions of health- care system functioning and their own knowledge? Gen Hosp Psychiatry 2017:46:88-93.

12 Liu C-F, Hedrick SC, Chaney EF, et al. Cost-effectiveness of collaborative care for depression in a primary care veteran population. Psychiatr Serv 2003;54:698-704.

13 Valentijn PP, Vrijhoef HJM, Ruwaard D, et al. Towards an international taxonomy of integrated primary care: a Delphi consensus approach. BMC Fam Pract 2015;16:64.

14 Rollman BL, Belnap BH, Mazumdar S, et al. A randomized trial to improve the quality of treatment for panic and generalized anxiety disorders in primary care. Arch Gen Psychiatry 2005;62:1332-41.

15 Roy-Byrne PP, Katon W, Cowley DS, et al. A randomized effectiveness trial of collaborative care for patients with panic disorder in primary care. Arch Gen Psychiatry 2001;58:869-76.

16 Morgan S, Pullon S, McKinlay E. Observation of interprofessional collaborative practice in primary care teams: an integrative literature review. Int J Nurs Stud 2015;52:1217-30.

17 Institute for Healthcare Improvement. Plan-Do-Study-Act (PDSA) Worksheet, 2020. Available: http://www.ihi.org/resources/Pages/ Tools/PlanDoStudyActWorksheet.aspx [Accessed 1 Jun 2019]. 